\title{
Preparation and properties of mesoporous silica/bismaleimide/diallylbisphenol composites with improved thermal stability, mechanical and dielectric properties
}

\author{
J. Hu, A. Gu*, G. Liang, D. Zhuo, L. Yuan \\ Department of Materials Science and Engineering, College of Chemistry, Chemical Engineering and Materials Science, \\ Soochow University, Suzhou, Jiangsu 215123, China
}

Received 2 November 2010; accepted in revised form 4 January 2011

\begin{abstract}
New composites with improved thermal stability, mechanical and dielectric properties were developed, which consist of 2,2'-diallylbisphenol A (DBA)/4,4'-bismaleimidodiphenylmethane (BDM) resin and a new kind of organic/inorganic mesoporous silica (MPSA). Typical properties (curing behavior and mechanism, thermal stability, mechanical and dielectric properties) of the composites were systematically investigated, and their origins were discussed. Results show that MPSA/DBA/BDM composites have similar curing temperature as DBA/BDM resin does; however, they have different curing mechanisms, and thus different crosslinked networks. The content of MPSA has close relation with the integrated performance of cured composites. Compared with cured DBA/BDM resin, composites with suitable content of MPSA show obviously improved flexural strength and modulus as well as impact strength; in addition, all composites not only have lower dielectric constant and similar frequency dependence, more interestingly, they also exhibit better stability of frequency on dielectric loss. For thermal stability, the addition of MPSA to DBA/BDM resin significantly decreases the coefficient of thermal expansion, and improves the char yield at high temperature with a slightly reduced glass transition temperature. All these differences in macro-properties are attributed to the different crosslinked networks between MPSA/DBA/BDM composites and DBA/BDM resin.
\end{abstract}

Keywords: thermosetting resins, bismaleimide, meosoporous silica, dielectric properties, structure-property

\section{Introduction}

Advancements in semiconductor miniaturization have propelled an increasing interest in high performance insulating materials, which are characterized with outstanding integrated properties including high mechanical properties, low dielectric constant and loss, good thermal stability, and desirable lower coefficient of thermal expansion (CTE). Thermosetting resins have gained considerable attention owing to their attractive processing characteristics and strong design-ability, so they are considered as the most promising materials for producing insulators $[1,2]$.

Bismaleimide (BMI), considered as a thermosetting polyimide, has been widespread used in various electronics and advanced technological applications. A few examples of these applications include radar and microwave structural composites, stealth technologies, space-ware composites, circuit boards, interconnects, and adhesives for microelectronics applications (e.g. flexible and rigid die-attach adhesives) [3-5]. However, its thermal stability, mechani-

\footnotetext{
*Corresponding author, e-mail: ajgu@suda.edu.cn
}

(c) BME-PT 
cal and dielectric properties need to be improved to meet the harsh requirements of future cutting-edge fields.

In fact, developing high performance insulators based on BMI resin has been one of the hottest topics in recent years. Some kinds of inorganic fillers have been used to prepare BMI resin matrix composites [6-9], of them mesoporous silica has attracted considerable interests owing to its highly ordered and uniform mesoporosity [10] as well as extremely low dielectric constant (1.4-2.1) [11-14]. However, present mesoporous silica mainly consists of $\mathrm{Si}-\mathrm{O}-\mathrm{Si}$ like zeolite, showing brittleness and poor interaction with organic polymers, and thus can not significantly improve the integrated performance of original polymers.

This article gives the first presentation of preparing new composites based on a new organic/inorganic mesoporous silica (MPSA) with functional $-\mathrm{NH}_{2}$ groups and 2,2'-diallylbisphenol A (DBA) modified 4,4'-bismaleimidodiphenylmethane (BDM) resin, and systematically investigating the influence of MPSA on the typical properties (curing behavior and mechanism, thermal stability, mechanical and dielectric properties) of composites and their origins. The aim of this article is not only to develop a new kind of high performance insulating composite, but also to provide a new example in evaluating the relationship of structure-property of advanced polymeric composites.

\section{Experimental}

\subsection{Materials}

4, 4'-Bismaleimidodiphenol methane (BDM) was obtained from Fengguang Chemical Co., Ltd. (China). 2, 2'-Diallylbisphenol A (DBA) was purchased from Laiyu Chemical Factory (China). $\gamma$ Aminopropyltriethoxysilane (analytical grade) was supplied by Jingzhou Jianghan Chemical Co., Ltd. (China). Tetraethoxysilane, tetramethylammonium hydroxide $5 \mathrm{H}_{2} \mathrm{O}$, methanol, dimethyldichlorosilane, hexane, and dimethylformamide were commercial products with analytical grades, they were purchased from Sinopharm Chemical Reagent Co., Ltd (China), and purified prior to use.

\subsection{Preparation of MPSA}

Cubic octameric silicate anion was prepared according to literature [15]. $20.8 \mathrm{~g}(0.1 \mathrm{~mol})$ tetraethoxysi- lane was added dropwise into a three-necked bottom flask containing $0.1 \mathrm{~mol}$ tetramethylammonium hydroxide and $91 \mathrm{ml}$ water. The reaction mixture was vigorously stirred at $23 \pm 2^{\circ} \mathrm{C}$ for $24 \mathrm{~h}$, and then $60^{\circ} \mathrm{C}$ for $10 \mathrm{~h}$. After that, the mixture was concentrated by distillation under reduced pressure. The concentrated product was cooled to $4{ }^{\circ} \mathrm{C}$, and then microcrystallites were precipitated from the cool solution. The crystal was separated by filtration, washed with acetone and dried in vacuum to afford cubic octameric silicate anion, $\left(\mathrm{Me}_{4} \mathrm{~N}\right)_{8} \mathrm{Si}_{8} \mathrm{O}_{20}$, coded as D4R.

5-10 ml methanolic solution of D4R (2 g) was added dropwise to a mixture of dimethyldichlorosilane $(2 \mathrm{ml})$, hexane $(30 \mathrm{ml})$ and dimethylformamide $(50 \mathrm{ml})$ with vigorous stirring in an ice bath. After the mixture was stirred for $1 \mathrm{~h}, 100 \mathrm{ml}$ water was added dropwise in $10 \mathrm{~min}$, followed by continuous stirring for $20 \mathrm{~min}$ at room temperature. After that, a hexane solution containing the resultant was separated, and hexane was removed at $60^{\circ} \mathrm{C}$ in vacuum, producing white solid (N-MPS) which was heated to $250^{\circ} \mathrm{C}$ in air and maintained at that temperature for $1.5 \mathrm{~h}$, and the resultant product is coded as MPS. $10 \mathrm{~g}$ MPS were added into $20 \mathrm{ml}$ ethanol solution of $\gamma$-aminopropyl triethoxy silane (1.5 g) with ultrasonic agitation for $30 \mathrm{~min}$ to form a homogenous suspension. Then distilled water $(2.22 \mathrm{~g})$ was poured into the suspension with magnetic stirring for $4 \mathrm{~h}$ at $50-60^{\circ} \mathrm{C}$. The suspension was separated by filtration, washed with ethanol and dried in air at about $100^{\circ} \mathrm{C}$. The resultant material was ground to pass through a 400 mesh sieve for analyses, and was coded as MPSA.

The structure of MPSA is illustrated in Figure 1 and Figure 2.

Solid-state ${ }^{29} \mathrm{Si}$ NMR of MPS (ppm): $-50.5\left(\mathrm{~T}_{2}\right.$, $\left.\mathrm{CH}_{3} \mathrm{Si}(\mathrm{OSi})_{2}\left(\mathrm{O}^{-}\right)\right),-60.7\left(\mathrm{~T}_{3}, \mathrm{CH}_{3} \mathrm{Si}(\mathrm{OSi})_{3}\right),-13.3$ $\left(\mathrm{D}_{2}, \mathrm{Si}\left(\mathrm{CH}_{3}\right)_{2}\left(\mathrm{O}^{-}\right)_{2}\right),-96.2\left(\mathrm{Q}_{3}, \mathrm{Si}(\mathrm{OSi})_{3}\left(\mathrm{O}^{-}\right)\right)$, $-104.6\left(\mathrm{Q}_{4}, \mathrm{Si}(\mathrm{OSi})_{4}\right)$. The existence of $\mathrm{T}_{2}, \mathrm{~T}_{3}$ and $\mathrm{Q}_{3}$ demonstrates that some $\mathrm{D}$ units in MPS are oxidized during the heat treatment.

FTIR of MPSA $\left(\mathrm{KBr}, \mathrm{cm}^{-1}\right)$ : 3440 (-OH stretching vibration), 2965 (C-H stretching vibration), 1265 ( $\mathrm{Si}-\mathrm{CH}_{3}$ deformation vibration), 1083 ( $\mathrm{Si}-\mathrm{O}-\mathrm{Si}$ stretching vibration), 1641 ( $-\mathrm{NH}_{2}$ deformation vibration), 850, 806 ( $\mathrm{Si}-\mathrm{CH}_{3}$ stretching vibration). The specific surface area of N-MPS is $22 \mathrm{~m}^{2} / \mathrm{g}$, while MPSA is $238 \mathrm{~m}^{2} / \mathrm{g}$, indicating that MPSA has 


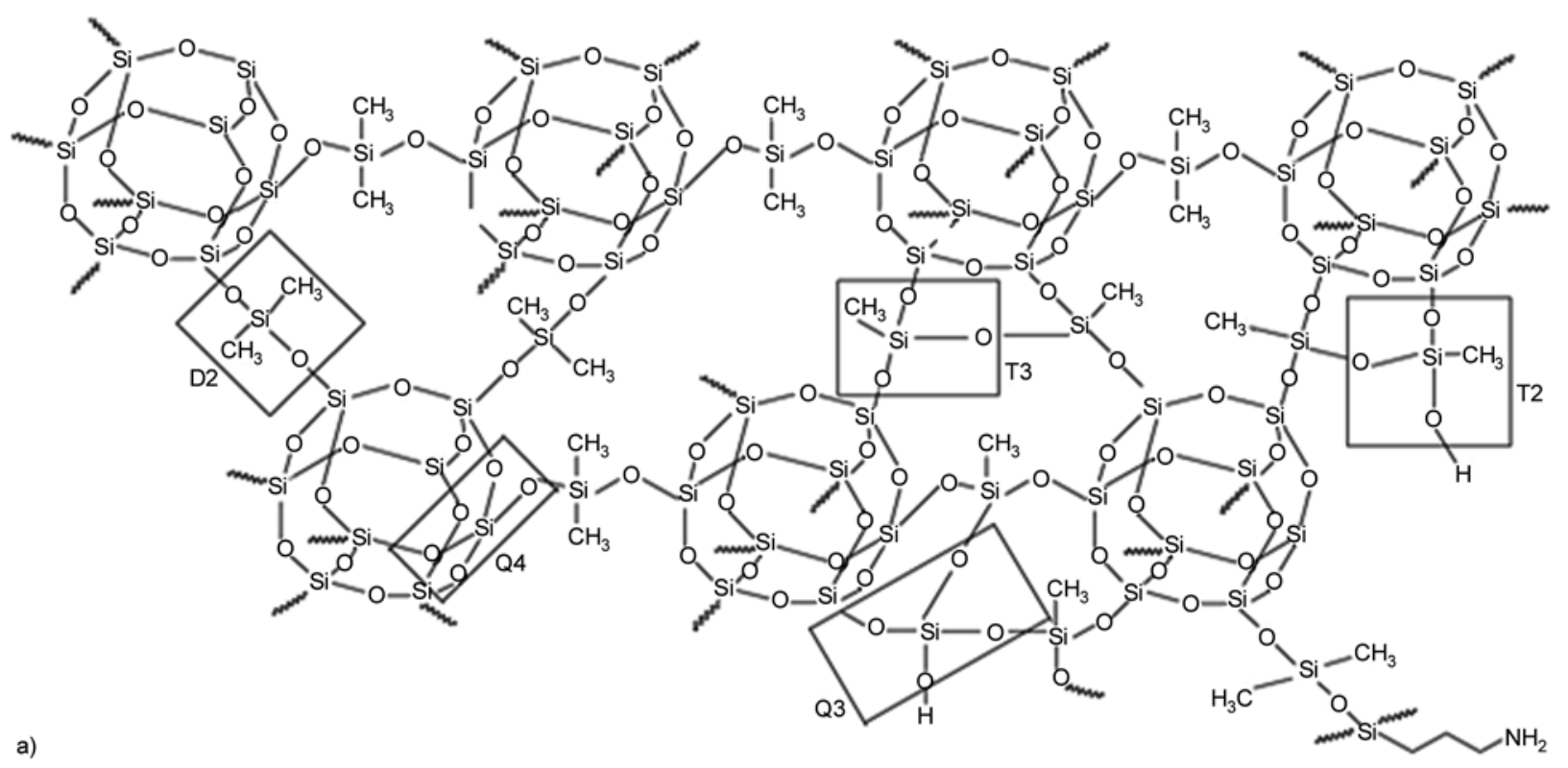

b)

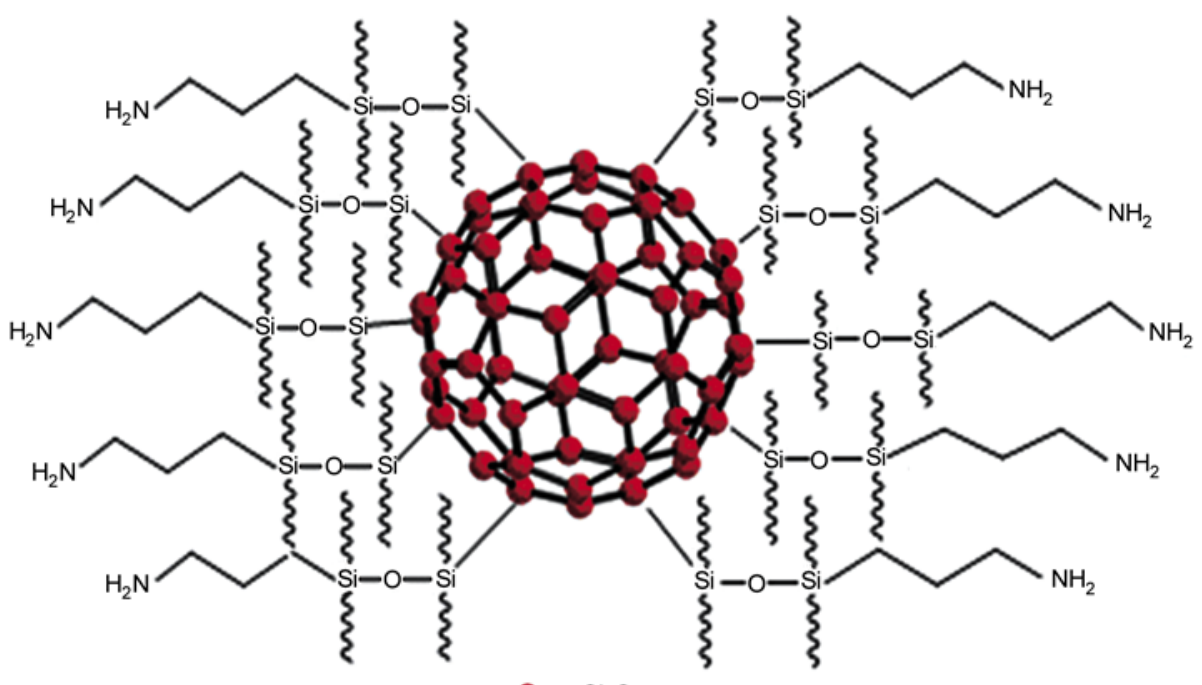

Figure 1. The chemical connection of MPSA (a), and chemical structure of MPSA (b)

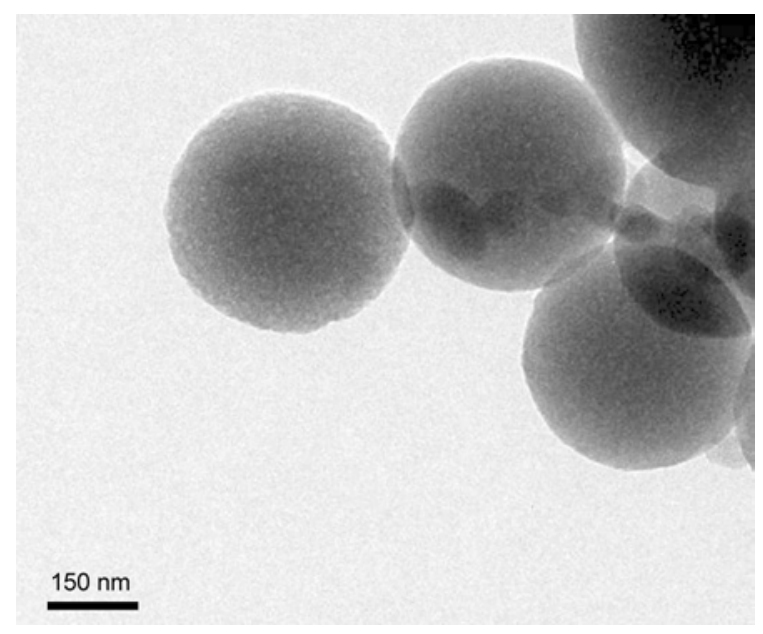

Figure 2. The TEM micrograph of MPSA a large amount of porosity. Some researchers have used similar method to demonstrate the existence of the 'porous' structure [14, 16-18].

\subsection{Preparation of MPSA/DBA/BDM composites}

Four formulations (Table 1) were evaluated to see the effect of differing stoichiometry of MPSA on the processing and performance parameters, and also to establish the formulational latitude this system provides.

Appropriate quantities of BDM and DBA were placed in a beaker with a mechanical stirrer and a thermometer. The mixture was heated to $130-135^{\circ} \mathrm{C}$, 
Table 1. Formulations and typical data from DSC analyses of DBA/BDM resin and MPSA/DBA/BDM composites

\begin{tabular}{|l|c|c|c|c|c|}
\hline \multicolumn{1}{|c|}{ Code } & $\begin{array}{c}\text { Weight ratio } \\
\text { DBA:BDM:MPSA }\end{array}$ & $\begin{array}{c}\mathbf{T}_{\mathbf{o n}} \\
{\left[{ }^{\circ} \mathbf{C}\right]}\end{array}$ & $\begin{array}{c}\mathbf{T}_{\text {exo }} \\
{\left[{ }^{\circ} \mathbf{C}\right]}\end{array}$ & $\begin{array}{c}\Delta \mathbf{H} \\
{[\mathbf{w} / \mathbf{g}]}\end{array}$ & $\begin{array}{c}\boldsymbol{\rho}\left(\mathbf{E}^{\prime}\right) \\
{\left[\mathbf{m o l} / \mathbf{m}^{3}\right]}\end{array}$ \\
\hline DBA/BDM & $82: 100: 0.00$ & 199 & 253 & 18.45 & 9492 \\
\hline MPSA1/DBA/BDM & $82: 100: 1.82$ & 193 & 252 & 19.31 & 10895 \\
\hline MPSA3/DBA/BDM & $82: 100: 5.46$ & 194 & 250 & 18.28 & 14418 \\
\hline MPSA5/DBA/BDM & $82: 100: 9.10$ & 199 & 251 & 17.52 & 22149 \\
\hline MPSA8/DBA/BDM & $82: 100: 14.56$ & 196 & 250 & 19.16 & 21919 \\
\hline
\end{tabular}

and maintained within that temperature range with stirring until a clear and brown liquid was obtained. The liquid was maintained at that temperature for additional 15 min to obtain a transparent liquid. Preweighted MPSA was added to the beaker slowly. After that, the mixture was kept at $130-135^{\circ} \mathrm{C}$ for 10min to obtain MPSA/DBA/BDM prepolymer.

Each prepolymer was thoroughly degassed at $120^{\circ} \mathrm{C}$, and poured into a preheated $\left(120^{\circ} \mathrm{C}\right)$ glass mold, then cured per the following curing procedure of $150^{\circ} \mathrm{C} / 2 \mathrm{~h}+180^{\circ} \mathrm{C} / 2 \mathrm{~h}+200^{\circ} \mathrm{C} / 2 \mathrm{~h}$. After that the cured sample was demolded and postcured in an air oven at $230^{\circ} \mathrm{C}$ for $4 \mathrm{~h}$. The resultant composite was coded as MPSA/DBA/BDM.

\subsection{Preparation of DBA/BDM resin}

Appropriate quantities of BDM and DBA were placed in a beaker with a mechanical stirrer and a thermometer. The mixture was heated to $130-135^{\circ} \mathrm{C}$ and maintained within that temperature range with stirring until a clear and brown liquid was obtained. The liquid was maintained at that temperature for additional $25 \mathrm{~min}$ to obtain a transparent liquid, which was DBA/BDM prepolymer.

Each prepolymer was thoroughly degassed at $120^{\circ} \mathrm{C}$ and poured into a preheated $\left(120^{\circ} \mathrm{C}\right)$ glass mold, then cured per the following curing procedure of $150^{\circ} \mathrm{C} / 2 \mathrm{~h}+180^{\circ} \mathrm{C} / 2 \mathrm{~h}+200^{\circ} \mathrm{C} / 2 \mathrm{~h}$. After that the cured sample was demolded and postcured in an air oven at $230^{\circ} \mathrm{C}$ for $4 \mathrm{~h}$. The resultant product was cured DBA/BDM resin.

\subsection{Measurements}

Solid-state ${ }^{29} \mathrm{Si}$ NMR spectrum was recorded using TMS as an internal standard on a UNITY INOVA400 (400 MHz NMR spectrometer, USA).

Fourier Transform Infrared (FTIR) spectra were recorded between 400 and $4000 \mathrm{~cm}^{-1}$ with a resolution of $2 \mathrm{~cm}^{-1}$ on a Nicolet FTIR 5700 spectrometer
(USA), and the sample powder was pressed into a pellet with $\mathrm{KBr}$.

Differential Scanning Calorimetry (DSC) analyses were done using DSC 200 F3 (NETZSCH, Germany) between 80 and $320^{\circ} \mathrm{C}$ at a heating rate of $10^{\circ} \mathrm{C} / \mathrm{min}$ in a nitrogen atmosphere.

Dynamic Mechanical Analysis (DMA) scans were performed in single-cantilever blending mode using a TA Instruments dynamic mechanical analyzer (DMA Q800, USA) from 50 to $350^{\circ} \mathrm{C}$ at a heating rate of $3^{\circ} \mathrm{C} / \mathrm{min}$ and a frequency of $1 \mathrm{~Hz}$.

Dielectric property was conducted over a frequency range from 10 to $10^{6} \mathrm{~Hz}$ at room temperature using a Broad Band Dielectric Spectrometer (Novocontrol Concept 80 Analyzer, Germany). The dimensions of each sample were $(25 \pm 0.2) \times(25 \pm 0.2) \times$ $(3 \pm 0.1) \mathrm{mm}^{3}$. The powders of MPSA were pressed into a pellet with a diameter of $20 \mathrm{~mm}$ and a thickness of $3 \mathrm{~mm}$.

Thermogravimetric (TG) analyses were performed using PerkinElmer TGA-7 (USA) at a heating rate of $10^{\circ} \mathrm{C} / \mathrm{min}$ in a nitrogen atmosphere from 60 to $780^{\circ} \mathrm{C}$. The initial decomposition temperature $\left(T_{\mathrm{di}}\right)$ was defined as the point of intersection at which the tangent of onset temperature and the tangent of the maximum degradation rate temperature.

Coefficient of Thermal Expansion (CTE) was measured using Diamond TMA (USA) from 60 to $270^{\circ} \mathrm{C}$. Each sample was dried to constant weight under $110^{\circ} \mathrm{C}$ before tests.

Flexural tests were done using a universal tester (China) according to GB2570-95. Impact strength was measured according to GB2571-95. At least five samples for each system were tested, and the average value was taken as the tested value.

The char residues of DBA/BDM resin and MPSA/ DBA/BDM composites were observed by an optical microscope (SMZ-T2, China).

The specific surface (BET) was performed with ASAP2020M (USA) automatic surface analyzer. 


\section{Results and discussion}

\subsection{Curing behavior of MPSA/DBA/BDM composites}

It is known that the curing behavior of a thermosetting resin determines its cross-linking network, and thereby the properties of the cured resin, so curing behavior is the first issue needs to be investigated on developing any thermosetting resin and related composites.

Figure 3 shows the DSC curves of DBA/BDM and MPSA/DBA/BDM prepolymers, each system shows one exothermic peak in the temperature range from 190 to $300^{\circ} \mathrm{C}$, the typical data such as the onset temperature $\left(T_{\mathrm{on}}\right)$, the maximum temperature of exothermic peaks $\left(T_{\text {exo }}\right)$, and the amount of exothermic heat $(\Delta H)$ obtained from these curves are summarized in Table 1. These results indicate that MPSA/ DBA/BDM systems have similar curing temperature and exothermic heat as DBA/BDM; hence the addition of MPSA to DBA/BDM resin does not change the curing profile of the resin. This conclusion can be confirmed from the FTIR spectra of MPSA/DBA/BDM systems with different curing processes as shown in Figure 4. For all systems, as the curing time and the curing temperature increase, the peak intensities of $\mathrm{C}=\mathrm{C}$ bonds at 3098, 1600, 1153,910 , and $686 \mathrm{~cm}^{-1}$ gradually decrease, and

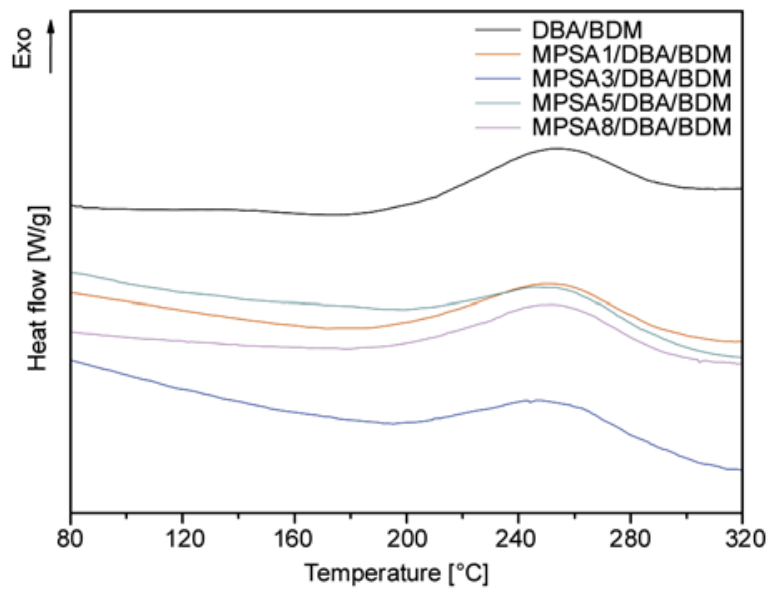

Figure 3. DSC curves of DBA/BDM and MPSA/DBA/ BDM prepolymers

Table 2. Coefficient $\mathrm{C}$ and $\mathrm{CTE}$ values of DBA/BDM and MPSA/DBA/BDM composites

\begin{tabular}{|l|c|c|}
\hline \multicolumn{1}{|c|}{ Sample } & Coefficient C & CTE $\left[\mathbf{p p m} /{ }^{\circ} \mathbf{C}\right]$ \\
\hline DBA/BDM & N/A & 93 \\
\hline MPSA1/DBA/BDM & 0.91 & 85 \\
\hline MPSA3/DBA/BDM & 0.70 & 75 \\
\hline MPSA5/DBA/BDM & 0.49 & 80 \\
\hline MPSA8/DBA/BDM & 0.52 & 90 \\
\hline
\end{tabular}

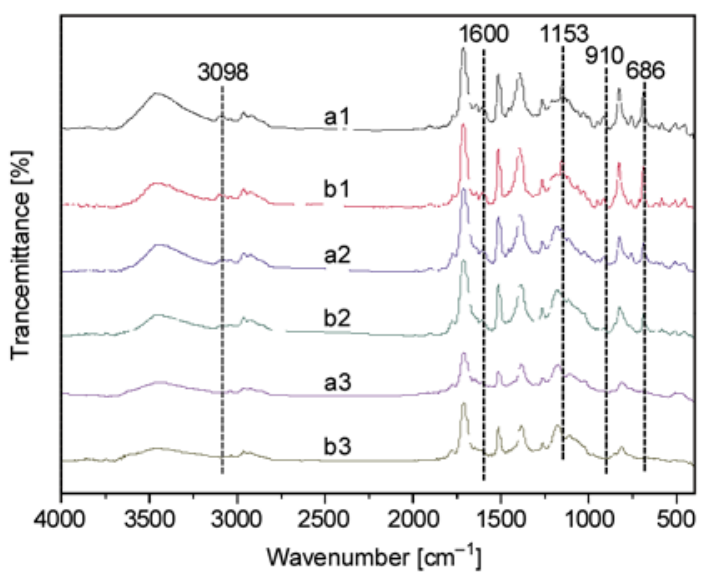

Figure 4. FTIR spectra of DBA/BDM (a) and MPSA5/ DBA/BDM (b) after being cured via different procedures $\left(1-\right.$ without curing; $2-150^{\circ} \mathrm{C} / 2 \mathrm{~h} ; 3-$ after postcured)

finally disappear, suggesting that under the curing and postcuring condition developed herein, the curing of all composites is complete [19].

However, the composites have different curing mechanism from DBA/BDM resin. For DBA/BDM resin, its curing mechanism contains multiple reactions, for example 'Ene' and 'Diels-Alder' reactions between maleimide group of BDM and allyl group of DBA, and the self-polymerization of BDM via $\mathrm{C}=\mathrm{C}$ bonds (Figure 5a) [20, 21]; while in the case of MPSA/DBA/BDM system, besides above reactions of $\mathrm{DBA} / \mathrm{BDM}$, there is a Michael reaction between maleimide and $-\mathrm{NH}_{2}$ groups [22]. This additional reaction not only supplies a chemical bonding between MPSA and DBA/BDM (Figure $5 b$ ) to guarantee a good dispersion of MPSA in the matrix, but also leads to a different structure of crosslinked network.

Specifically, the effect of MPSA on the crosslinked structure is complicated, because MPSA is an organic/inorganic hybrid containing flexible $\mathrm{Si}-\mathrm{O}-\mathrm{Si}$ bonds and aliphatic chains, rigid $\mathrm{SiO}_{2}$ with cage structure as well as functional $-\mathrm{NH}_{2}$ groups. First, because the Michael reaction between BDM and $-\mathrm{NH}_{2}$ takes place at lower temperature than the homopolymerization of BDM does, so the addition of MPSA to DBA/BDM resin will produce flexible segments, and decrease the amount of the rigid polybismaleimide. Second, although the content of MPSA is small, there are a lot of $-\mathrm{NH}_{2}$ groups on the surface of MPSA, meaning that the presence of MPSA tends to shorten the distance between thecrosslinking points, and thus increases the crosslink- 
<smiles>C=CCc1cc(PC)ccc1O</smiles><smiles>CC(C)N1C(=O)C2c3c(P)ccc(O)c3CC(CC3CC(=O)N(C)C3=O)C2C1=O</smiles>

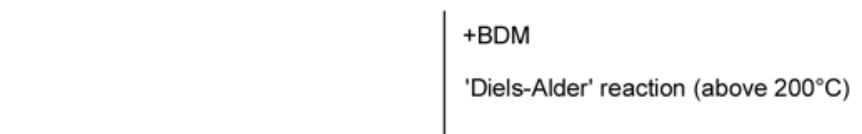

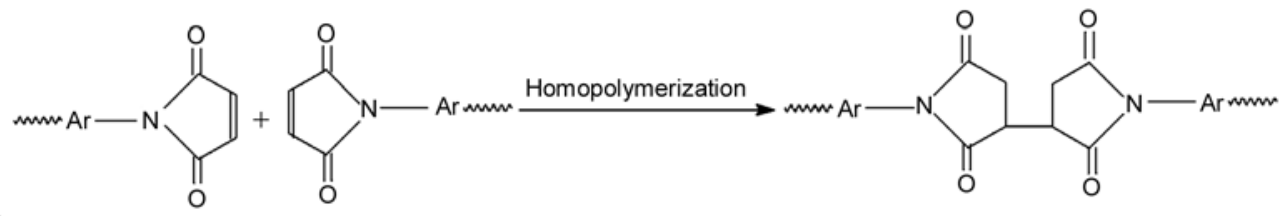

a)

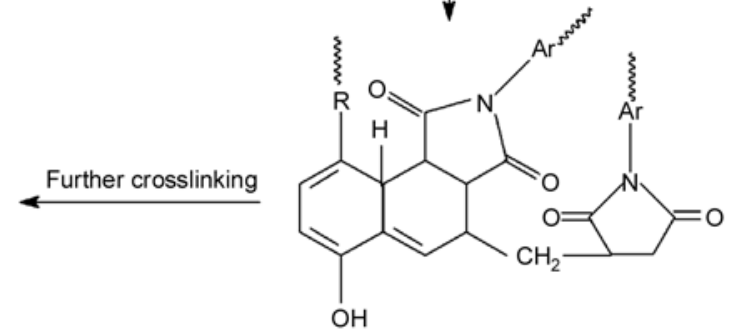

b)<smiles>CN(N1C(=O)C[CH+]CC1=O)N1C(=O)CC(NCCCSOP(C)(C)(C)O)C1=O</smiles>

Figure 5. The chemical reactions among BDM, DBA (a) and MPSA (b)

ing density $\left(\rho_{\left(\mathrm{E}^{\prime}\right)}\right)$ of the resultant network. Third, the cage units of MPSA connect with each other to form a compact network, as one part of the whole crosslinked network of composites, tending to improve the crosslinking density of the composites. In order to confirm the effect of MPSA on the crosslink density, the $\rho_{\left(\mathrm{E}^{\prime}\right)}$ values of DBA/BDM resin and MPSA/DBA/BDM composites were calculated. Generally, the $\rho_{\left(\mathrm{E}^{\prime}\right)}$ values of a highly crosslinked polymer and related particle filled composites can be calculated by the classical equation based on the statistical theory of rubber elasticity [23] as shown in Equation (1):

$$
\rho_{\left(E^{\prime}\right)}=\frac{G^{\prime}}{3 \Phi R T}
$$

where $G^{\prime}$ is the storage modulus (Figure 6) of the sample at the temperature $T$ from DMA analyses; $\Phi$ is the front factor, and assumed to be 1 [23]; $T$ is the absolute temperature at which the sample is in rubbery state, herein $T$ is selected as the temperature which is $40^{\circ} \mathrm{C}$ higher than the glass transition temperature; $R$ is the gas constant.

According to Equation (1), the calculated $\rho_{\left(\mathrm{E}^{\prime}\right)}$ values of cured DBA/BDM resin and MPSA/DBA/ BDM composites were summarized in Table 1. It can be seen that all composites have bigger crosslink 


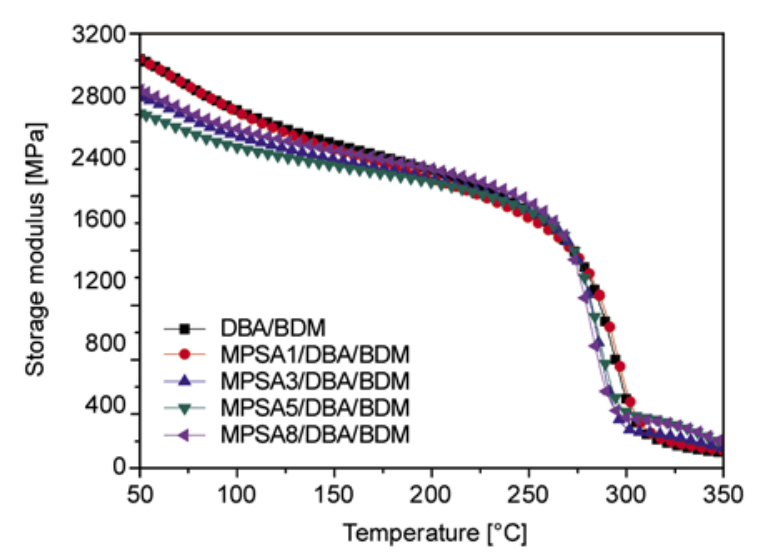

Figure 6. Storage moduli of cured DBA/BDM resin and MPSA/DBA/BDM composites

densities than DBA/BDM resin; moreover, with increasing the content of MPSA, the crosslink density of composite initially increases and then levels off.

\subsection{Mechanical properties of MPSA/DBA/BDM composites}

Flexural property is usually used for evaluating the mechanical properties of a material because the flexural loading is very complicated and may contain different types of loadings such as tensile, shearing and/or compressive loads [24]. Figure 7 shows the flexural properties of cured DBA/BDM resin and MPSA/DBA/BDM composites. With a small addition of MPSA to DBA/BDM resin, both flexural strength and modulus increase, and then reach their respective maximum values at about $3 \mathrm{wt} \%$ of MPSA. Specifically, the maximum flexural strength and modulus are $187 \mathrm{MPa}$ and $4.5 \mathrm{GPa}$, respectively, which are about 20 and 10\% higher than the corresponding values of DBA/BDM resin. Further increas-

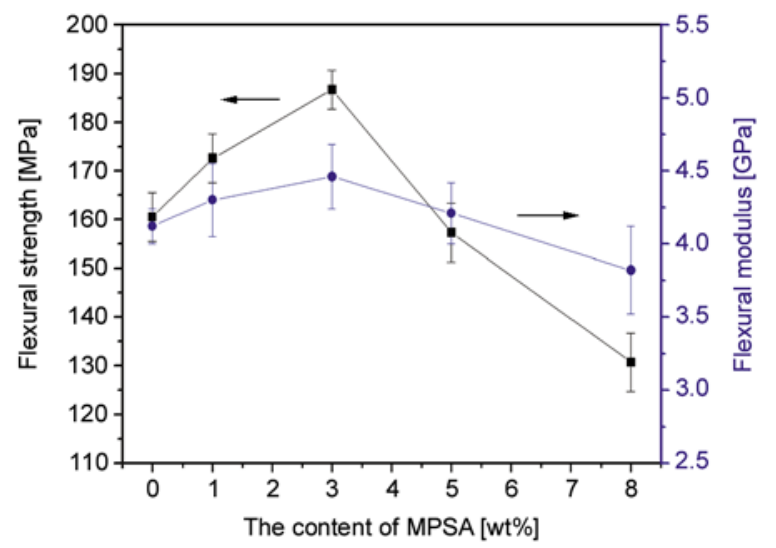

Figure 7. Flexural strengths and moduli of cured DBA/ $\mathrm{BDM}$ resin and MPSA/DBA/BDM composites ing the content of MPSA, both flexural strength and modulus of composites decrease.

Although some mesoporous silica/polymer composites have been reported [14, 25], the majority of them are in the form of films; hence their flexural properties were almost not investigated. With regard to polyhedral oligomeric silsesquioxane (POSS)/ polymer composites, only several pieces of literature report the flexural properties. Liang's group prepared POSS/cyanate ester nanocomposites, and found that the flexural modulus of the nanocomposite with $3 \mathrm{wt} \%$ POSS is about $15 \%$ higher than that of neat cyanate ester resin, while the composite has the similar flexural strength as the resin does [26]. Li's group also reported similar results in the POSS/ epoxy nanocomposites [27]. However, Rashid's group reported a different phenomenon [28], in detail, the addition of POSS to epoxy resin can obviously increase the flexural strength, but does not change the flexural modulus. Comparing these results in literature with the data in this paper, an interesting but preliminary conclusion could be stated that MPSA which consists of a large amount of POSS units and organic chains maybe more effective in improving both flexural strength and modulus than single POSS. A reasonable mechanism is not clear, which is worthy to be intensively investigated.

It is well known that the flexural strength of a material reflects the rigidity and toughness of the material, so those factors which are beneficial to improve the stiffness and/or the toughness can improve the flexural strength. Flexural modulus reflects the coinherent energy of a material, and the ability to resist strain, so a thermosetting resin with bigger molecular weight and a larger amount of rigid structure generally has higher modulus [29]. For a welldispersed particle/thermosetting resin composite, the crosslinking density is an important factor of determining the stiffness and toughness, and thus has significant influence on the flexural strength; in addition, the interfacial adhesion determines the stress transfer under an external loading, and thus provides additional contribution to the flexural properties.

As for MPSA/DBA/BDM composites, the co-reaction between MPSA and DBA/BDM resin supplies good interfacial adhesion, and a large amount of aminopropyl chains grafted on the surface of MPSA are expected to constitute a rough particle surface, 
which may give rise to a distinct structural difference between the interfacial zone and the bulk, and thus further improve the strength and modulus of MPSA/DBA/BDM composites [30, 31]. In addition, compared with cured DBA/BDM resin, MPSA/DBA/ BDM composites have larger crosslink density, leading to the increment of flexural strength and modulus. In detail, the Michael reaction between maleimide and $-\mathrm{NH}_{2}$ groups in composites decreases the amount of the rigid homopolymer of BDM, resulting in decreased flexural properties. Note that the Michael reaction also introduces flexible $\mathrm{Si}-\mathrm{O}-\mathrm{Si}$ chains into the crosslinked network, leading to increased flexural strength but decreased modulus. Obviously these opposite influences play a combined role on the flexural properties, and therefore there is an optimum content of MPSA to get the highest flexural strength and modulus.

Figure 8 shows the impact strengths of cured DBA/ $\mathrm{BDM}$ resin and MPSA/DBA/BDM composites with different contents of MPSA, it can be seen that the impact strength of MPSA/DBA/BDM composites is closely dependent on the content of MPSA, following the similar trend of flexural properties. Specifically, there exists an optimum content of MPSA to obtain the biggest impact strength, that is, the impact strengths of MPSA3/DBA/BDM is $17.2 \mathrm{~kJ} / \mathrm{m}^{2}$, about 1.4 times of that of DBA/BDM resin.

Similar increment of impact strength owing to the addition of rod-like attapulgite to polyimide resin is observed [30], which is considered as the particle toughening effect. Some other models including crack pinning, crack bridging, and debonding of particles as well as crack deflection have been proposed to explain this effect $[32,33]$.

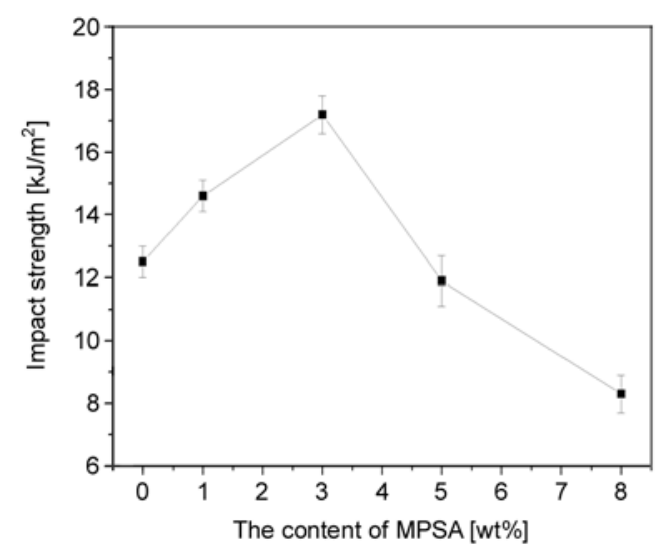

Figure 8. Impact strengths of cured DBA/BDM resin and MPSA/DBA/BDM composites
Besides, two opposite influences are also responsible for the effect of the content of MPSA on the impact strength. In detail, the introduced flexible $\mathrm{Si}-\mathrm{O}-\mathrm{Si}$ chains and decreased amount of rigid homopolymer of BDM are beneficial to increase the impact strength; while oppositely, the increased crosslinking density will decrease the impact strength. As a result, the impact strength of MPSA/ DBA/BDM composites exhibit strong composition dependence.

The coefficient $(C)$ is generally used to describe the effectiveness of fillers on the modulus of composite, which can be calculated by Equation (2) [34]:

$C=\frac{\left(\frac{E_{\mathrm{G}}^{\prime}}{E_{\mathrm{R}}^{\prime}}\right)_{\text {composite }}}{\left(\frac{E_{\mathrm{G}}^{\prime}}{E_{\mathrm{R}}^{\prime}}\right)_{\text {resin }}}$

where $E_{\mathrm{G}}^{\prime}$ and $E_{\mathrm{R}}^{\prime}$ are the storage moduli in the glassy and rubbery regions from DMA tests, respectively.

For MPSA/DBA/BDM composites developed herein, the modulus of each composite at 60 or $320^{\circ} \mathrm{C}$ is regarded as $E_{\mathrm{G}}^{\prime}$ or $E_{\mathrm{R}}^{\prime}$, respectively. Table 2 gives the calculated coefficient $C$ values of all MPSA/DBA/ BDM composites, because a small $C$ value reflects a big effectiveness of fillers on the modulus of composite, so it can be concluded that the composition of MPSA/DBA/BDM composites has obvious effect on the $C$ value. The composite with larger content of MPSA tends to have a smaller $C$ value, that is, with the increase of the content of MPSA, the $C$ value obviously decreases; however when the content of MPSA is bigger than $5 \mathrm{wt} \%$, the $C$ value almost levels off.

From the viewpoint of molecular structure, the intensity of the storage modulus below $T_{\mathrm{g}}$ reflects the rigidity of materials. Figure 6 shows that the intensities of storage moduli of MPSA/DBA/BDM composites in glassy state are lower than that of DBA/BDM resin, this phenomenon is different from the common inorganic filler/polymer composites [35], indicating that MPSA is not a rigid filler owing to the presence of a large amount of organic chains between POSS structures as well as the porous. 


\subsection{Dielectric properties}

As described above, one important reason to synthesize and employ MPSA for developing high performance insulators is that MPSA is expected to have extremely low dielectric constant and loss owing to its unique porous structure. Figures 9 and 10 confirm this expectation, so it is easy to understand that the addition of MPSA to DBA/BDM resin decreases the dielectric constant over the whole frequency range, and the larger is the content of MPSA, the lower is the dielectric constant; however, this trend does not fit MPSA8/DBA/BDM composite. Specifically, the magnitude of dielectric constant follows the order of DBA/BDM $\approx$ MPSA8/ DBA/BDM $>$ MPSA1/DBA/BDM $>$ MPSA3/DBA/ $\mathrm{BDM}>\mathrm{MPSA}$ /DBA/BDM. This is because with the increase of the content of MPSA, the amount of the flexible chains resulting from the Michael reaction between BDM and MPSA increases, at the same time, the amount of the rigid polybismaleimide

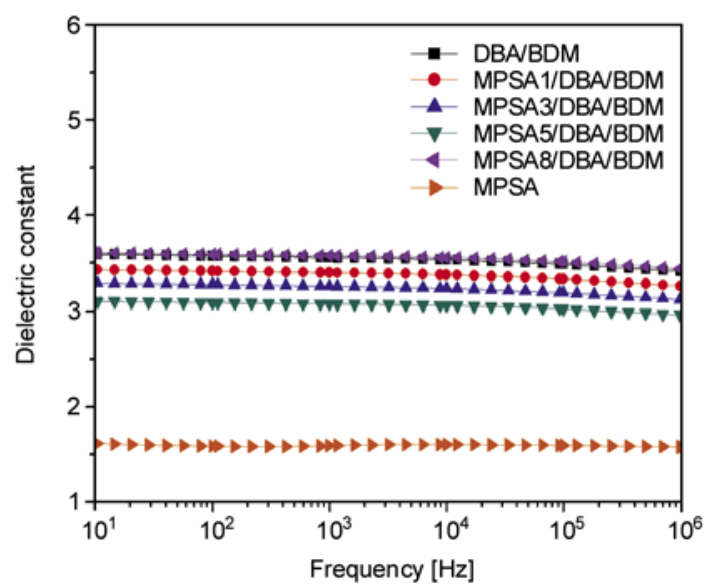

Figure 9. Dependence of dielectric constant on frequency of cured DBA/BDM resin and MPSA/DBA/ BDM composites

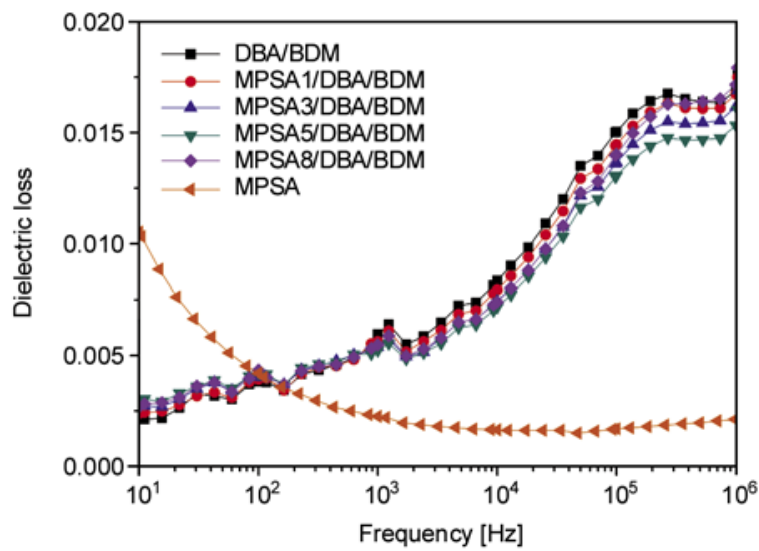

Figure 10. Dependence of dielectric loss on frequency of cured DBA/BDM resin and MPSA/DBA/BDM composites decreases, so the polarization and relaxation of dipoles are easy to occur, and thus increase the dielectric constant and loss. This influence enhances with the increase of the content of MPSA.

For the binary composites, its dielectric constant can be calculated by Equation (3) [36]:

$\log \varepsilon_{\mathrm{c}}=Y_{1} \cdot \log \varepsilon_{1}+Y_{2} \cdot \log \varepsilon_{2}$

where $\varepsilon_{\mathrm{c}}, \varepsilon_{1}$, and $\varepsilon_{2}$ stand for the dielectric constant of MPSA/DBA/BDM composites, DBA/BDM resin and MPSA at $10 \mathrm{~Hz}$, respectively; $Y_{1}$ and $Y_{2}$ represent the volume fraction of DBA/BDM resin and MPSA, respectively.

The theoretical dielectric constants calculated by Equation (3) and the experimental data of MPSA/ DBA/BDM composites are shown in Figure 11. There is obvious deviation between theoretical and experimental values, and the larger is the content of MPSA, the bigger is the deviation. This is because that Equation (3) is developed without considering the interfacial effect on the dielectric constant of a composite. A good interfacial adhesion is beneficial to restrict the polarization and relaxation of dipoles, so it is not surprise to find that the dielectric constant of MPSA/DBA/BDM composites has a deviation from the values calculated by Equation (3), and the composite with larger content of MPSA has bigger difference. In addition, it is expected that MPSA/ DBA/BDM composites have lower dielectric constant than the values calculated from Equation (3). However, MPSA8/DBA/BDM composite does not follow this expectation again, owing to the reason described above.

The dependence of dielectric loss on frequency for $\mathrm{DBA} / \mathrm{BDM}$ resin and all composites is shown in

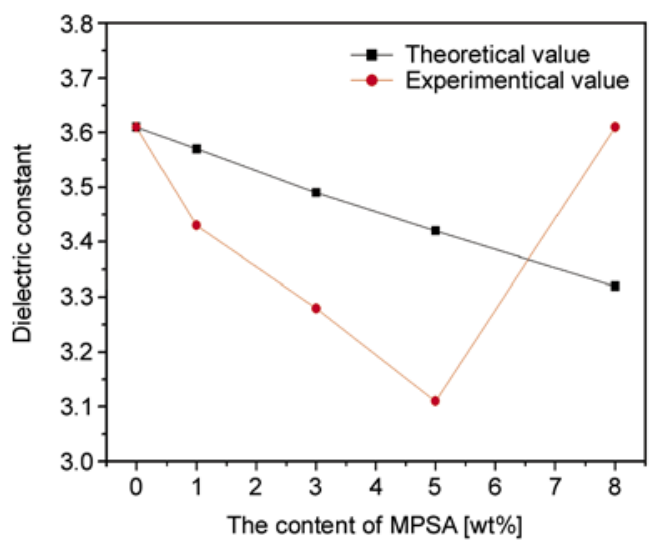

Figure 11. Theoretical and experimental dielectric constants of cured DBA/BDM resin and MPSA/DBA/ BDM composites 
Figure 10. The dielectric loss of DBA/BDM resin increases with the increase of frequency because the molecule movement can not follow up the variety of frequency of the applied electric field [37]; while that of MPSA shows opposite trend owing to its special structure. MPSA is a kind of organic/ inorganic mesoporous silica, the existence of porous structure will lead to big space charge polarization and thus big dielectric loss at low frequency. Therefore, the resultant composites exhibit a combined feature of their separate components. Compared with DBA/BDM resin, MPSA/DBA/BDM composites have slightly bigger dielectric loss at low frequency, and obviously smaller values at high frequency, indicating that these composites have better frequency stability of dielectric loss. This is an important and attractive merit for developing high performance insulators.

\subsection{Thermal properties}

Heat-resistance of a material can be reflected by many properties, among them the glass transition temperature $\left(T_{\mathrm{g}}\right)$, TG behavior and CTE are three most important aspects for high performance insulators.

Several methods can be used to detect the $T_{\mathrm{g}}$ value of a polymer, however, DMA technique is the most effective one [34]. The peak temperature of loss modulus-temperature plot is defined as the glass transition temperature herein. As shown in Figure 12 that DBA/BDM resin shows a sharp peak with a shoulder at higher temperature. By Gaussian fitting [38], each curve can be divided into two peaks, one small peak appears at $296^{\circ} \mathrm{C}$, and another big peak appears at $305^{\circ} \mathrm{C}$, indicating that $\mathrm{DBA} / \mathrm{BDM}$ resin has a multi-phase structure, and thus two $T_{\mathrm{g}}$ values. According to the thermal stability, the first damping peak of cured DBA/BDM resin at the lower temperature could be attributed to the $T_{\mathrm{g}}$ of the addition product resulted from the 'Ene' and 'Diels-Alder' reactions between maleimide groups of BDM and

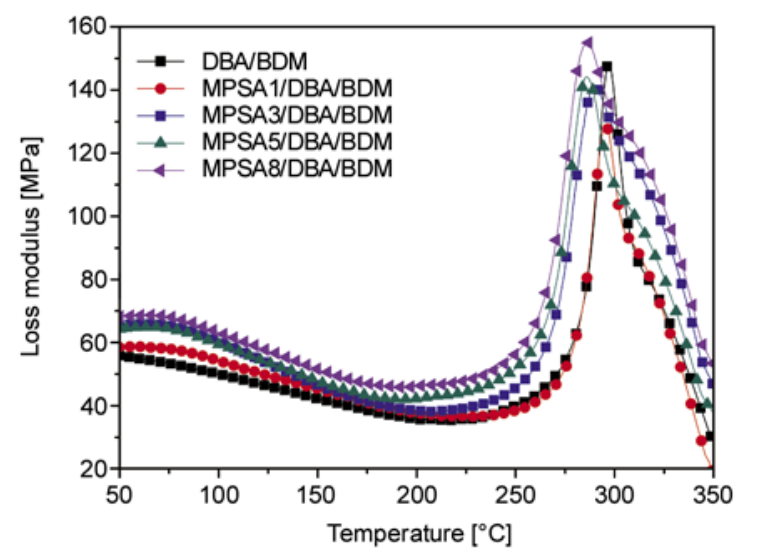

Figure 12. Overlay plots of loss modulus-temperature for cured DBA/BDM resin and MPSA/DBA/BDM composites

allyl groups of DBA; while the second damping peak at higher temperature might be assigned to the homopolymer of BDM and further crosslinked structure between BDM and DBA [19].

MPSA/DBA/BDM composites exhibit similar shapes to those of the DBA/BDM resin, but the first damping peak slightly shifts toward lower temperature, while the second damping peak almost does not change. Obviously, the curing mechanism of composites results in this phenomenon. As discussed above, the curing mechanism of MPSA/DBA/BDM composites not only includes the curing reactions taking place in the DBA/BDM resin, but also contains additional co-reaction between $-\mathrm{NH}_{2}$ groups on MPSA and maleimide groups of BDM, forming flexible $\mathrm{Si}-\mathrm{O}-\mathrm{Si}$ chains, and thus lower $T_{\mathrm{g}}$ value; while oppositely, interfacial confinement effect is conducive to improve the $T_{\mathrm{g}}$ value. Obviously, the above two aspects play a combined role. In addition, the multi-reactions are also responsible for the broadened shapes of damping peaks for composites.

The thermogravimetric behavior can be reflected by TG analyses, Figure 13 shows the TG and DTG curves of all samples, the typical data such as the initial decomposition temperature $\left(T_{\mathrm{di}}\right)$, the temperature of the maximum degradation rate $\left(T_{\max }\right)$, and

Table 3. Characteristic data from TG analyses of DBA/BDM and MPSA/DBA/BDM composites

\begin{tabular}{|c|c|c|c|c|c|}
\hline \multirow{2}{*}{ Resin } & \multirow{2}{*}{$\begin{array}{c}\mathbf{T}_{\mathbf{d i}} \\
{\left[{ }^{\circ} \mathbf{C}\right]}\end{array}$} & \multirow{2}{*}{$\begin{array}{l}\mathbf{T}_{\max } \\
{\left[{ }^{\circ} \mathbf{C}\right]}\end{array}$} & \multirow{2}{*}{$\begin{array}{c}\text { Intensity } \\
{\left[\% /{ }^{\circ} \mathrm{C}\right]}\end{array}$} & \multicolumn{2}{|c|}{$Y_{\mathrm{c} \text { at } 780^{\circ} \mathrm{C}}[\%]$} \\
\hline & & & & Theoretical & Experimental \\
\hline DBA/BDM & 410 & 450 & 0.67 & 29.1 & 29.1 \\
\hline MPSA1/DBA/BDM & 413 & 449 & 0.57 & 30.0 & 31.8 \\
\hline MPSA3/DBA/BDM & 410 & 449 & 0.50 & 31.7 & 40.7 \\
\hline MPSA5/DBA/BDM & 409 & 449 & 0.48 & 33.4 & 44.3 \\
\hline MPSA8/DBA/BDM & 410 & 449 & 0.46 & 35.9 & 46.1 \\
\hline MPSA & 465 & $534 / 754$ & $0.04 / 0.07$ & 86.1 & 86.1 \\
\hline
\end{tabular}




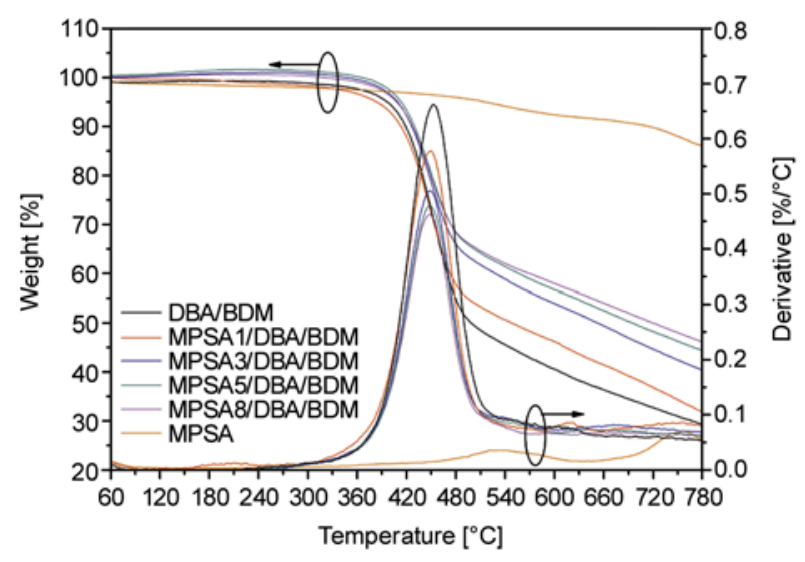

Figure 13. TG and DTG curves of cured DBA/BDM resin and MPSA/DBA/BDM composites

char yield $\left(Y_{\mathrm{c}}\right)$ at $780^{\circ} \mathrm{C}$ obtained from these curves are summarized in Table 3. All composites have similar $T_{\mathrm{di}}$ and $T_{\max }$ values as DBA/BDM resin, indicating that they have similar thermal degradation mechanism; however, the peak intensity of DBA/ BDM resin is significantly higher than that of MPSA/ DBA/BDM composites, suggesting that the thermal decomposition is delayed because of the presence of MPSA. It is worth noting that the experimental $Y_{\mathrm{c}}$ of composites is much larger than that of DBA/ $\mathrm{BDM}$ resin, and even more interestingly, the former is bigger than its theoretical value calculated by the 'Mixture Rule', and this phenomenon is enhanced as the content of MPSA increases, demonstrating that there is a synergistic effect in the composites which is believed to come from the interaction between MPSA and the matrix described above.

Figure 14 shows the micrographs of char residues after TG tests. The char residue of DBA/BDM resin shows loose structure; while that of each composite is dense, and the composite with a larger content of MPSA has denser char residue. That is because a large amount of MPSA will form more amount of inert silica layer, which can act as a thermal barrier to inhibit further degradation of the inner part of the matrix [39]. That is also the reason why the composite with a larger content of MPSA has bigger experimental $Y_{\mathrm{c}}$ than theoretical value.

CTE is a very important property in actual applications, which denotes the dimension stability of a material with the variation of service temperature. For example, heat is produced when a microelectronic product (especially that with high performance) works, so organic resins with a low CTE are needed to reduce the mismatch in thermal expan- sion among different materials in the product, otherwise the product tends to deform during the service, and may lead to break down [28].

CTE values of DBA/BDM resin and MPSA/DBA/ $\mathrm{BDM}$ composites over a temperature range from $60-270^{\circ} \mathrm{C}$ are summarized in Table 2. It is interesting to find that all MPSA/DBA/BDM composites have lower CTE values than DBA/BDM resin. In detail, CTE values of MPSA/DBA/BDM composites are related to the content of MPSA. Specifically, with the initial increase of the content of MPSA, the CTE value decreases, and reaches the minimum value at $3 \mathrm{wt} \%$ MPSA; further increasing the content of MPSA, the CTE value gradually increases.

The decreased CTE value for composites is attributed to a combined influence consisting in three aspects. First, the interfacial interaction between MPSA and DBA/BDM resin constrains the movement of the polymer network due to the presence of covalently bonded polymer layers, and thus leads to decreased CTE values; however, second, which also decreases the amount of the homopolymer of BDM, and thus tends to increase the CTE value. Third, the decrement in nanoparticle dispersion at larger loadings, and the effect of decreased interparticle distance which gives rise to the additional loss in configurational entropy at the interface and in density fluctuation in the zone surrounding nanoparticles [40] also increase the CTE value; Obviously, the determining aspect varies with the content of MPSA, the former plays the dominate role when the content of MPSA is small, while the latter becomes the key one when the content of MPSA is big.

\section{Conclusions}

A new organic/inorganic mesoporous silica (MPSA) is used to develop new high performance bismaleimide/diallylbisphenol A resin matrix composites with improved mechanical and dielectric properties as well as thermal resistance.

The addition of MPSA to DBA/BDM resin does not change the curing profile of DBA/BDM resin, but varies the curing mechanism, leading to different crosslinked networks, and thus the integrated performance of cured composites. Apparently, the content of MPSA exhibits a great influence on the integrated performance of cured composites. The outstanding integrated performance of MPSA/DBA/ BDM composites with suitable content of MPSA 


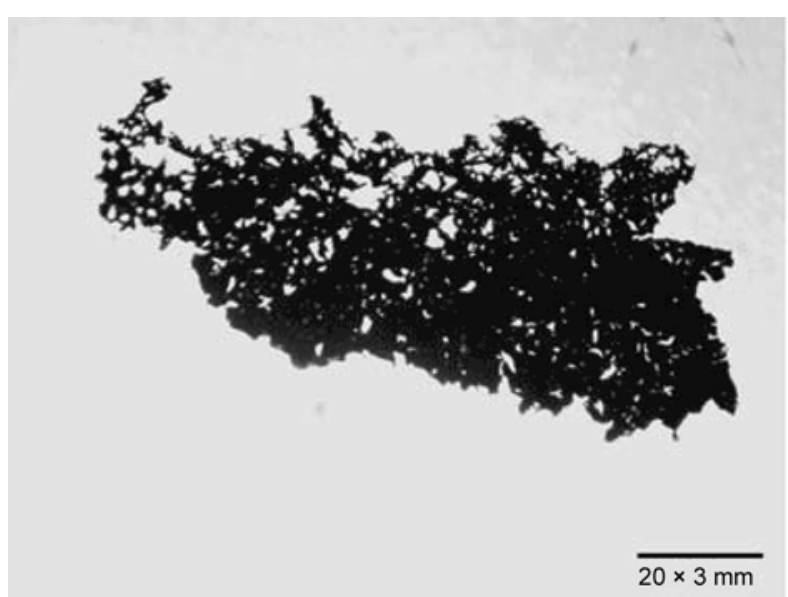

a)

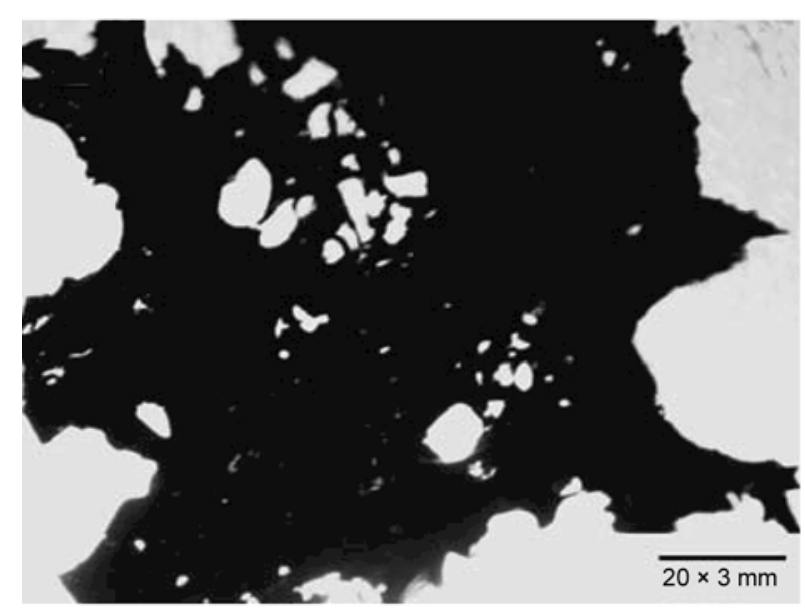

c)

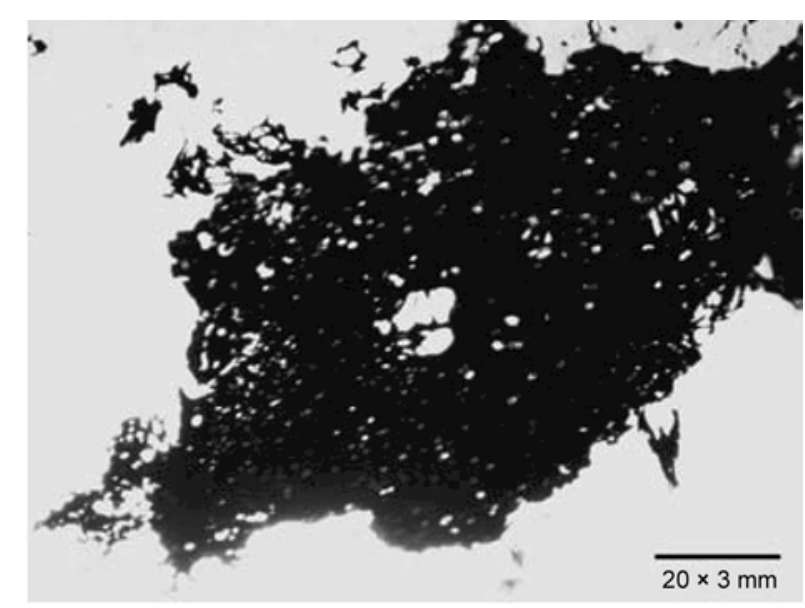

b)

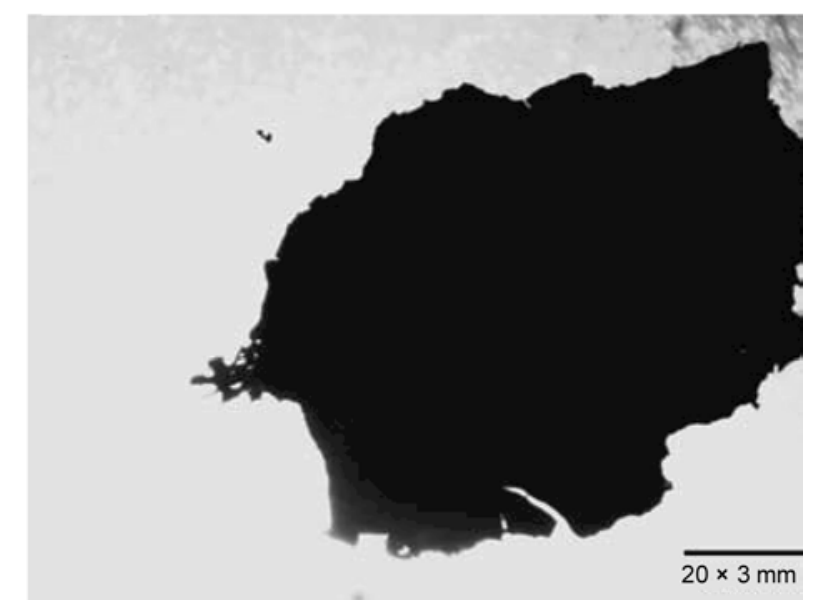

d)

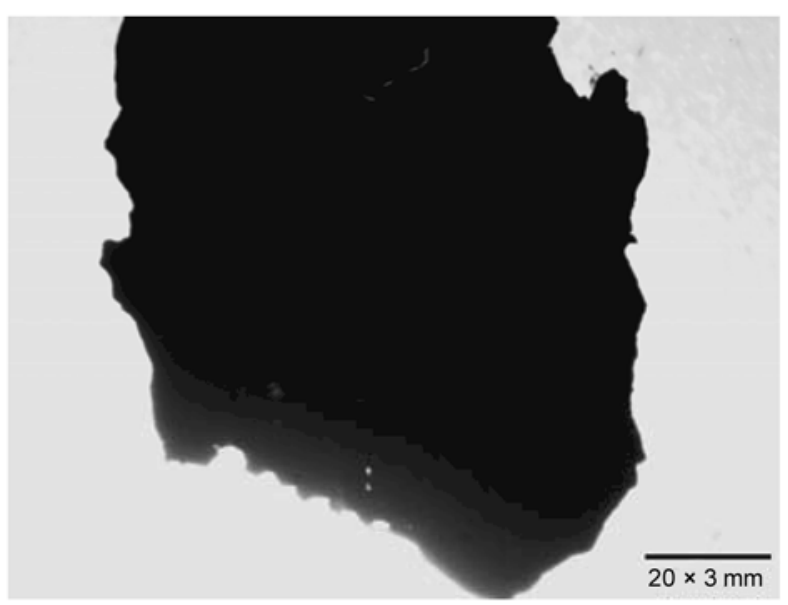

e)

Figure 14. Optical pictures of the char residues of cured DBA/BDM resin (a) and MPSA/DBA/BDM composites (b: MPSA1/DBA/BDM, c: MPSA3/DBA/BDM, d: MPSA5/DBA/BDM, e: MPSA8/DBA/BDM)

show a great potentiality to be used as high performance insulators for applications needing harsh requirements in thermal, mechanical and dielectric properties.

\section{Acknowledgements}

The authors thank 'Qing Lan Project' (2008), '333 Talent Project' (2008) and 'Six Talent Peaks' (2009) of Jiangsu Province, and National Natural Science Foundation of China (20974076) for financially supporting this project. 


\section{References}

[1] Ahner N., Schulz S. E., Blaschta F., Rennau M.: Thermal stability and gap-fill properties of spin-on MSQ low-k dielectrics. Microelectronic Engineering, 84, 2606-2609 (2007).

DOI: 10.1016/j.mee.2007.06.007

[2] Johnson M., Li Z. J., Wang J. L., Yan Y. S.: Mechanical characterization of zeolite low dielectric constant thin films by nanoindentation. Thin Solid Films, 515, 3164-3170 (2007).

DOI: $10.1016 /$ j.tsf.2006.01.048

[3] Rajasekaran R., Alagar M., Karikal Chozhan C.: Effect of polyethersulfone and $N, N^{\prime}$-bismaleimido4,4'-diphenyl methane on the mechanical and thermal properties of epoxy systems. Express Polymer Letters, 2, 339-348 (2008).

DOI: $10.3144 /$ expresspolymlett.2008.40

[4] Fan S. L., Boey F. Y. C., Abadie M. J. M.: UV curing of a liquid based bismaleimide-containing polymer system. Express Polymer Letters, 1, 397-405 (2007). DOI: $10.3144 /$ expresspolymlett.2007.56

[5] Yan H-Q., Wang H-Q., Cheng J.: Interpenetrating polymer networks from the novel bismaleimide and cyanate containing naphthalene: Cure and thermal characteristics. European Polymer Journal, 45, 23832390 (2009).

DOI: 10.1016/j.eurpolymj.2009.04.031

[6] Lu H. B., Shen H. B., Song Z. L., Shing K. S., Tao W., Nutt S.: Rod-like silicate-epoxy nanocomposites. Macromolecular Rapid Communications, 26, 1445 1450 (2005).

DOI: $10.1002 /$ marc. 200500360

[7] Rao Y. Q., Pochan J. M.: Mechanics of polymer-clay nanocomposites. Macromolecules, 40, 290-296 (2007). DOI: $10.1021 / \mathrm{ma} 061445 \mathrm{w}$

[8] Liang G. Z., Hu X. L.: Aluminum-borate-whiskersreinforced bismaleimide composites. 1: Preparation and properties. Polymer International, 53, 670-674 (2004).

DOI: $10.1002 /$ pi.1401

[9] Yuan L., Ma X. Y., Gu A. J., Yan H. X., Liang G. Z., Wang W., Wu J. Y.: A novel organic rectorite modified bismaleimide/diallylbisphenol A system. Polymers for Advanced Technologies, 20, 826-833 (2009). DOI: $10.1002 /$ pat.1328

[10] Park I., Peng H-G., Gidley D. W., Xue S. Q., Pinnavaia T. J.: Epoxy-silica mesocomposites with enhanced tensile properties and oxygen permeability. Chemistry of Materials, 18, 650-656 (2006).

DOI: $10.1021 / \mathrm{cm} 051768 \mathrm{r}$

[11] Baskaran S., Liu J., Domansky K., Kohler N., Li X. H., Coyle C., Fryxell G. E., Thevuthasan S., Williford R. E.: Low dielectric constant mesoporous silica films through molecularly templated synthesis. Advanced Materials, 12, 291-294 (2000).

DOI: 10.1002/(SICI)1521-4095(200002)12:4<291:: AID-ADMA291>3.0.CO;2-P
[12] Yang C. M., Cho A. T., Pan F. M., Tsai T. G., Chao K. J.: Spin-on mesoporous silica films with ultralow dielectric constants, ordered pore structures, and hydrophobic surfaces. Advanced Materials, 13, 10991102 (2001).

DOI: 10.1002/1521-4095(200107)13:14<1099::AIDADMA1099>3.0.CO;2-0

[13] Zhao D. Y., Yang P. D., Melosh N., Feng J. L., Chmelka B. F., Stucky G. D.: Continuous mesoporous silica films with highly ordered large pore structures. Advanced Materials, 10, 1380-1385 (1998).

DOI: 10.1002/(SICI)1521-4095(199811)10:16<1380:: AID-ADMA1380>3.0.CO;2-8

[14] Lin J. J., Wang X. D.: Preparation, microstructure, and properties of novel low- $\kappa$ brominated epoxy/mesoporous silica composites. European Polymer Journal, 44, 1414-1427 (2008).

DOI: $10.1016 /$ j.eurpolymj.2008.02.022

[15] Hagiwara Y., Shimojima A., Kuroda K.: Alkoxysilylated-derivatives of double-four-ring silicate as novel building blocks of silica-based materials. Chemistry of Materials, 20, 1147-1153 (2008).

DOI: $10.1021 / \mathrm{cm} 0716194$

[16] Hasegawa I.: Building block approach to organic/silica hybrid materials. Journal of Sol-Gel Science and Technology, 5, 93-100 (1995).

DOI: $10.1007 / \mathrm{BF} 00487725$

[17] Ho K. Y., McKay G., Yeung K. L.: Selective adsorbents from ordered mesoporous silica. Langmuir, 19, 3019-3024 (2003). DOI: $10.1021 / 1 \mathrm{la0267084}$

[18] Suzuki N., Kiba S., Yamauchi Y.: Low dielectric property of novel mesoporous silica/polymer composites using smart molecular caps: Theoretical calculation of air space encapsulated inside mesopores. Microporous and Mesoporous Materials, 138, 123-131 (2011). DOI: $10.1016 /$ j.micromeso.2010.09.020

[19] Gouri C., Reghunadhan Nair C. P., Ramaswamy R.: Reactive Alder-ene blend of diallyl bisphenol A novolac and bisphenol A bismaleimide: Synthesis, cure and adhesion studies. Polymer International, 50, 403-413 (2001).

DOI: $10.1002 /$ pi.644

[20] Gu A. J., Liang G. Z.: High performance bismaleimide resins modified by novel allyl compounds based on epoxy resins. Polymer-Plastics Technology and Engineering, 36, 681-694 (1997). DOI: $10.1080 / 03602559708000654$

[21] Rozenberg B. A., Dzhavadyan E. A., Morgan R., Shin E.: High-performance bismaleimide matrices: Cure kinetics and mechanism. Polymers for Advanced Technologies, 13, 837-844 (2002).

DOI: $10.1002 /$ pat.230 
[22] Liang K. W., Toghiani H., Li G. Z., Pittman Jr C. U.: Synthesis, morphology, and viscoelastic properties of cyanate ester/polyhedral oligomeric silsesquioxane nanocomposites. Journal of Polymer Science Part A: Polymer Chemistry, 43, 3887-3898 (2005).

DOI: $10.1002 /$ pola.20861

[23] Kimura H., Matsumoto A., Sugito H., Hasegawa K., Ohtsuka K., Fukuda A.: New thermosetting resin from poly(p-vinylphenol) based benzoxazine and epoxy resin. Journal Applied Polymer Science, 79, 555-565 (2001).

DOI: $10.1002 / 1097-4628(20010118) 79: 3<555:: A I D-$ APP190>3.0.CO;2-H

[24] Song H. C.: Polymer composites. Beijing University of Aeronautics and Astronautics Press, Beijing (1985).

[25] Lin J. J., Wang X. D.: Novel low-א polyimide/mesoporous silica composite films: Preparation, microstructure, and properties. Polymer, 48, 318-329 (2007). DOI: 10.1016/j.polymer.2006.10.037

[26] Liang K. W., Li G. Z., Toghiani H., Koo J. H., Pittman Jr C. U.: Cyanate ester/polyhedral oligomeric silsesquioxane (POSS) nanocomposites: Synthesis and characterization. Chemistry of Materials, 18, 301-312 (2006). DOI: $10.1021 / \mathrm{cm} 051582 \mathrm{~s}$

[27] Li G. Z., Wang L. C., Toghiani H., Daulton T. L., Koyama K., Pittman Jr C. U.: Viscoelastic and mechanical properties of epoxy/multifunctional polyhedral oligomeric silsesquioxane nanocomposites and epoxy/ ladderlike polyphenylsilsesquioxane blend. Macromolecules, 34, 8686-8693 (2001).

DOI: $10.1021 / \mathrm{ma} 011117 \mathrm{q}$

[28] Rashid E. S. A., Ariffin K., Kooi C. C., Akil H. M.: Preparation and properties of POSS/epoxy composites for electronic packaging applications. Material and Design, 30, 1-8 (2009).

DOI: $10.1016 /$ j.matdes.2008.04.065

[29] Karthikeyan C. S., Sankaran S., Kishore: Investigation of bending modulus of fiber-reinforced syntactic foams for sandwich and structural applications. Polymers for Advanced Technologies, 18, 254-256 (2007). DOI: $10.1002 /$ pat.828

[30] Li A., Pan Y. Z., Shen X. W., Lu H. B., Yang Y. L.: Rod-like attapulgite/polyimide nanocomposites with simultaneously improved strength, toughness, thermal stability and related mechanisms. Journal of Materials Chemistry, 18, 4928-4941 (2008).

DOI: $10.1039 / \mathrm{B} 805849 \mathrm{~K}$
[31] Starr F. W., Douglas J. F., Glotzer S. C.: Origin of particle clustering in a simulated polymer nanocomposite and its impact on rheology. The Journal of Chemical Physics, 119, 1777-1789 (2003).

DOI: $10.1063 / 1.1580099$

[32] Pan Y. Z., Xu Y., Li A., Lu H. B., Yang Y. L., Chen W., Nutt S.: Hybrid network structure and mechanical properties of rodlike silicate/cyanate ester nanocomposites. Macromolecules, 41, 9245-9258 (2008). DOI: $10.1021 / \mathrm{ma} 800819 \mathrm{~s}$

[33] Johnsen B. B., Kinloch A. J., Mohammed R. D., Taylor A. C., Sprenger S.: Toughening mechanisms of nanoparticle-modified epoxy polymers. Polymer, 48, 530-541 (2007).

DOI: 10.1016/j.polymer.2006.11.038

[34] Ling W., Gu A. J., Liang G. Z., Yuan L., Liu J.: Dynamic mechanical properties of aluminum nitride/ cyanate ester composites for high performance electronic packaging. Polymers for Advanced Technologies, 21, 365-370 (2010).

DOI: $10.1002 /$ pat. 1436

[35] Omrani A., Afsar S., Safarpour M. A.: Thermoset nanocomposites using hybrid nano $\mathrm{TiO}_{2}-\mathrm{SiO}_{2}$. Materials Chemistry and Physics, 122, 343-349 (2010). DOI: $10.1016 /$ j.matchemphys.2010.02.073

[36] Malini K. A., Mohammed E. M., Sindhu S., Joy P. A., Date S. K., Kulkarni S. D., Kurian P., Anantharaman M. R.: Magnetic and processability studies on rubber ferrite composites based on natural rubber and mixed ferrite. Materials Science, 36, 5551-5557 (2001). DOI: 10.1023/A:1012545127918

[37] Hu G. J., Hong X. K., Sun J. L., Chen J., Chu J. H., Zhu D-M., Dai N.: Peculiar ferroelectric and dielectric properties of quasiperiodic $\mathrm{PbZr}_{0.4} \mathrm{Ti}_{0.6} \mathrm{O}_{3}$ multilayers. New Journal Physics, 8, 316-324 (2006). DOI: $10.1088 / 1367-2630 / 8 / 12 / 316$

[38] Stephen M. A., Granick S.: Image analysis with rapid and accurate two-dimensional Gaussian fitting. Langmuir, 25, 8152-8160 (2009).

DOI: $10.1021 / 1 a 900393 \mathrm{v}$

[39] Zhang Z. P., Gu A. J., Liang G. Z., Ren P. G., Xie J. Q., Wang X. L.: Thermo-oxygen degradation mechanisms of POSS/epoxy nanocomposites. Polymer Degradation and Stability, 92, 1986-1993 (2007).

DOI: $10.1016 /$ j.polymdegradstab.2007.08.004

[40] Rittigstein P., Priestley R. D., Broadbelt L. J., Torkelson J. M.: Model polymer nanocomposites provide an understanding of confinement effects in real nanocomposites. Nature Materials, 6, 278-282 (2007).

DOI: $10.1038 /$ nmat1870 